clearance (milliliters per minute) was calculated as dose - $\alpha \cdot 10^{3} / \mathrm{A}$, where dose is expressed in milligrams, $\mathrm{A}$ is the intercept of the clearance curve on the concentration axis (expressed in milligrams per liter), and $\alpha$ (per minute $)=\log 2 / \mathrm{T}^{1 / 2}$, where $\mathrm{T}^{1 / 2}$ is the clearance halftime (expressed in minutes). This calculation assumes that the hepatic extraction efficiency of ICG is essentially $100 \% .^{4}$

Mean cardiac output was just above baseline levels during CPB in all four groups of patients, and it increased by a further $6 \%$ to $25 \%$ after CPB (Table I). During normothermic CPB, mean liver blood flow did not change significantly in either the pulsatile group (14\% mean decrease) or the nonpulsatile group ( $6 \%$ mean decrease); immediately after CPB, liver blood flow was significantly higher than baseline only in the patients receiving nonpulsatile perfusion (Table II). During hypothermic CPB, mean liver blood flow decreased significantly compared with baseline in the pulsatile group ( $30 \%$ mean decrease) but not in the nonpulsatile group (13\% mean decrease).

We conclude that hepatic blood flow is well maintained by both pulsatile and nonpulsatile flow during normothermic CPB at $2.4 \mathrm{~L} \cdot \mathrm{min}^{-1} \cdot \mathrm{m}^{-2}$. However, a decrease in liver blood flow during hypothermic perfusion is associated more with pulsatile than with nonpulsatile flow. These data support the previously published reports from clinical ${ }^{2}$ and animal ${ }^{3}$ investigations, which noted preservation of liver blood flow during hypother- mic, nonpulsatile flow, together with loss of benefit owing to pulsatility at 2.4 compared with $1.2 \mathrm{~L} \cdot \mathrm{min}^{-1}$ $\cdot \mathrm{m}^{-2}$. They are also consistent with our recent clinical report of reduced gastric mucosal perfusion during hypothermic CPB. ${ }^{5}$ The present findings thus support the view that an optimum CPB protocol for preserving the hepatic circulation requires a high perfusion rate with pulsatile or nonpulsatile flow at $37^{\circ} \mathrm{C}$; if hypother$\mathrm{mia}$ is used, a nonpulsatile protocol appears to be preferable to a pulsatile one.

\section{REFERENCES}

1. Hampton WW, Townsend, MC, Schirmer WJ, Haybron DM, Fry DE. Effective hepatic blood flow during cardiopulmonary bypass. Arch Surg 1989;124:458-9.

2. Yamada T, Nomoto S, Aota M, Nishimura K, Matsuda K, Ban T. Hepatic circulation during nonpulsatile cardiopulmonary bypass. ASAIO J 1995;41:M294-7.

3. Desai JB, Mathie RT, Taylor KM. Hepatic blood flow during cardiopulmonary bypass in the dog: the effect of temperature, flow rate and pulsatility. Perfusion 1993;8:149-58.

4. Grainger SL, Keeling PW, Brown IM, Marigold JH, Thompson RP. Clearance and non-invasive determination of the hepatic extraction of indocyanine green in baboons and man. Clin Sci 1983;64:207-12.

5. Mathie RT, Ohri SK, Keogh BE, Williams J, Siney L, Griffith TM. Nitric oxide activity in patients undergoing cardiopulmonary bypass. J Thorac Cardiovasc Surg 1996;112:1394-5.

\title{
METHEMOGLOBINEMIA COMPLICATING TOPICAL ANESTHESIA DURING BRONCHOSCOPIC PROCEDURES
}

\author{
Bryan Clary, MD, ${ }^{a}$ Lynne Skaryak, MD, ${ }^{a}$ Mark Tedder, MD, ${ }^{a}$ Andrew Hilton, MD, ${ }^{\mathrm{b}}$ Gregory Botz, MD, ${ }^{\mathrm{b}}$ and

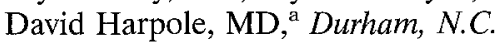

Methemoglobinemia is a potential complication of topical anesthesia during bronchoscopic procedures. If unrecognized, it can lead to death. The presentations and management of two patients in whom methemoglobinemia developed after topical anesthesia with benzocaine during treatment with a flexible bronchoscope are presented, followed by a brief review of the literature.

From the Departments of Surgery ${ }^{a}$ and Anesthesiology, ${ }^{b}$ Duke University Medical Center, Durham, N.C.

Received for publication Dec. 20, 1996; accepted for publication Jan. 30, 1997.

Address for reprints: Bryan M. Clary, MD, Box 3324 DUMC, Durham, NC 27710.

J Thorac Cardiovase Surg 1997;114:293-5

Copyright (C) 1997 by Mosby-Year Book, Inc.

$0022-5223 / 97 \$ 5.00+0 \quad \mathbf{1 2 / 5 4 / 8 0 7 7 7}$

\section{Clinical summaries}

PATIENT 1. A 67-year-old man underwent resection of a posterior mediastinal mass. On postoperative day 2 a chest roentgenogram showed complete opacification of the left lung field and a leftward shift of the mediastinal structures, consistent with a collapse of the left lung. A fiberoptic bronchoscope was inserted at the bedside with the aid of topical anesthesia. The nasopharynx was lubricated with viscous lidocaine and the oropharynx was sprayed twice with a $20 \%$ benzocaine solution (Hurricaine Spray). Bronchoscopic examination revealed significant mucus plugging of the left main-stem bronchus. Twenty minutes into the procedure the patient was noticed to be cyanotic. The oxygen saturation by pulse oximetry $\left(\mathrm{Spo}_{2}\right)$ had dropped gradually from $94 \%$ at the beginning of the procedure to $60 \%$ to $65 \%$. The patient became disoriented and was promptly intubated. The initial arterial blood gas value immediately after intubation with the 
patient receiving an inspired oxygen fraction of 1.0 revealed an oxygen tension of $271 \mathrm{~mm} \mathrm{Hg}$ with a calculated oxygen saturation of $100 \%$, despite the deeply cyanotic appearance of the blood sample. The methemoglobin level was analyzed by co-oximetry and determined to be $35.4 \%$ with a measured oxygen saturation of $67.7 \%$. Methylene blue dye $(2 \mathrm{mg} / \mathrm{kg})$ was administered intravenously as a $1 \%$ solution over 10 minutes. Within 20 minutes after administration of the dye, the $\mathrm{Spo}_{2}$ had returned to normal. The patient was extubated without difficulty the next day after an uneventful second bronchoscopic examination for which intratracheal $4 \%$ lidocaine was used as the anesthetic.

PATIENT 2. Two weeks after patient 1 was treated, a 66-year-old man with a history of coronary artery disease underwent a pulmonary resection (right upper lobectomy), decortication, and pleural stripping for a $3 \mathrm{~cm}$ large-cell carcinoma with associated necrotizing pneumonia. On postoperative day 2 the patient was noted to have significant atelectasis of the right middle lobe. A fiberoptic bronchoscope was inserted at the bedside with the aid of topical anesthesia. The nasopharynx was lubricated with viscous lidocaine and the oropharynx was sprayed twice with a $20 \%$ benzocaine solution (Hurricaine Spray). The procedure, which lasted only 5 minutes, revealed minimal secretions and no significant mucus plugs. Ten minutes after the termination of the procedure, the patient's $\mathrm{Spo}_{2}$ began to steadily decline to $60 \%$. The patient's mental status did not change significantly, and he had no symptoms of respiratory distress. The patient was given oxygen by means of a face mask and an arterial blood gas sample was drawn. The arterial oxygen tension from this deeply cyanotic sample was $347 \mathrm{~mm} \mathrm{Hg}$. A methemoglobin level could not be obtained because the in-hospital co-oximeter was not functioning. The patient was empirically given an intravenous dose of methylene blue dye, $2 \mathrm{mg} / \mathrm{kg}$, and within 30 minutes the $\mathrm{Spo}_{2}$ had normalized.

Discussion. The flexible bronchoscope is commonly used for both diagnostic and therapeutic purposes by the busy thoracic surgeon. The introduction of the flexible fiberoptic bronchoscope by Ikeda in 1967 presented the thoracic surgeon with a valuable new diagnostic instrument. The instrument can be easily passed through the nose or mouth of an awake patient after the brief application of a topical anesthetic. Methemoglobinemia arising from topical anesthesia with benzocaine is a potential complication that, if unrecognized, can lead to death. Knowledge of and immediate attention to this complication are critical in managing these patients. Although reports have surfaced describing this reaction during endotracheal intubation during anesthesia and in patients undergoing endoscopic examination of the upper part of the intestine, its occurrence during use of a flexible bronchoscope has not been widely reported in the thoracic surgical literature.

Methemoglobin is formed when the heme iron of unoxygenated hemoglobin is oxidized to the ferric $\left(\mathrm{Fe}^{3+}\right)$ state. This is to be characterized from the physiologic equilibrium of oxygenated hemoglobin that exists between the ferric and ferrous states in relation to the capture and release of oxygen. Methemoglobin is not capable of carrying oxygen or carbon dioxide. In the erythrocyte, an equilibrium normally exists between hemoglobin and methemoglobin. In normal circumstances, only $1 \%$ of hemoglobin within the erythrocyte exists in the met form. The term methemoglobinemia refers to levels of methemoglobin greater than $1 \%$. Normal levels of methemoglobin are maintained by two mechanisms: (1) the presence of reductive metabolic pathways that regulate the amount of oxidant substances which can oxidize the heme iron to the ferric state and (2) directly reducing the ferric iron state of methemoglobin to the ferrous state. The more important regulation is by conversion of methemoglobin to hemoglobin. This reduction of methemoglobin is carried out by the enzymes NADH methemoglobin reductase and NADPH methemoglobin reductase, with the former accounting for approximately $95 \%$ of reducing activity in vivo. Although enzymatic reduction of methemoglobin by NADPH methemoglobin reductase accounts for only $5 \%$ of the total reducing activity, this reaction is greatly accelerated by the presence of methylene blue, which acts as a cofactor.

Acquired hemoglobinemia occurs when the rate of formation of methemoglobin exceeds the rate of reduction as a result of exposure to certain substances: amyl nitrite, aniline dyes, benzocaine, bismuth subnitrate, dapsone, lidocaine, nitroglycerin, $p$-aminosalicylic acid, phenytoin, prilocaine, primaquine, pyridine, silver nitrate, and sulfonamides. Nitrites and aniline derivatives are the chemicals most commonly associated with methemoglobinemia. The toxic effects of benzocaine appear to arise from the oxidizing capabilities of a metabolite that is most likely an $N$-hydroxy derivative. Animal and human data suggest that the formation of clinically significant methemoglobinemia after exposure to benzocaine is limited to a small subgroup of patients. Differences in benzocaine metabolism may explain the variability in benzocaineassociated methemoglobinemia. ${ }^{1}$ Lidocaine, which is also commonly used during bronchoscopic studies, has been demonstrated to cause methemoglobinemia. As demonstrated by the first patient, lidocaine can be used in patients sensitive to benzocaine although reports of methemoglobinemia occurring after lidocaine anesthesia in these patients exist. ${ }^{2}$ Data within the existing literature are not sufficient to comment on the relative propensity of benzocaine versus lidocaine in inducing methemoglobinemia.

The clinical effects of methemoglobinemia derive from the blood's decreased oxygen-carrying capacity. Symptoms include anxiety, headaches, fatigue, coma, and death and usually occur when the methemoglobin level exceeds $30 \% .^{3}$ When levels exceed $50 \%$ tissue oxygenation becomes truly inadequate, resulting in dyspnea, acidosis, bradycardia, paralysis, coma, and convulsions. Death usually occurs with levels above $70 \%{ }^{3}$ Cyanosis is usually evident when the methemoglobin level is $10 \%$ to $15 \%$, although in anemic individuals it can appear with levels of $2.5 \%$. The diagnosis of methemoglobinemia after topical anesthesia with benzocaine during bronchoscopic studies is suggested by the appearance of chocolate-colored cyanosis and an oxygen-unresponsive drop in $\mathrm{SpO}_{2}$ as measured by pulse oximetry. Reports in the literature have suggested that the $\mathrm{Spo}_{2}$ with increasing levels of methemoglobin plateaus at $85 \% .^{4}$ We observed $\mathrm{SpO}_{2}$ levels far 
below this. Thus levels below $85 \%$ should not exclude the diagnosis of methemoglobinemia. The arterial blood sample despite this low $\mathrm{Spo}_{2}$ will reveal a normal or even elevated oxygen tension in the patient being provided oxygen. Qualitative measurement of methemoglobin levels are obtained with co-oximetry.

The treatment of methemoglobinemia begins with general supportive care, including oxygen and control of the airway if necessary. Methylene blue given in a dose of 1 to $2 \mathrm{mg} / \mathrm{kg}$ intravenously over 10 minutes is the treatment of choice. Methylene blue is reduced to leukomethylene blue by accepting electrons from NADPH in the presence of NADPH methemoglobin reductase. Leukomethylene blue then acts as an electron donor and nonenzymatically reduces methemoglobin to hemoglobin. ${ }^{3}$ In higher doses, methylene blue can produce side effects including chest pain, dyspnea, tremors, and exacerbation of methemoglobinemia by directly oxidizing hemoglobin to methemoglobin. Methemoglobin levels after administration of methylene blue return to normal levels within 20 minutes to 1 hour. If cyanosis persists beyond 1 hour, a second dose may be given. In severe or refractory cases, exchange transfusion may be used. Patients unresponsive to methylene blue treatment may have a congenital deficiency in either glucose-6-phosphate dehydrogenase or NADPH methemoglobin reductase.
In summary, benzocaine-induced methemoglobinemia should be considered in patients undergoing bronchoscopic study when cyanosis develops that is unresponsive to oxygen and associated with normal to high oxygen tensions. Diagnostic confirmation by means of co-oximetry if available should be performed. Intravenous methylene blue is the treatment of choice in patients who have symptomatic levels or who are at high risk for cardiovascular events. Its use is contraindicated in patients with known glucose-6-phosphate dehydrogenase deficiency. Exchange transfusion should be considered in symptomatic patients with near lethal levels.

\section{REFERENCES}

1. Guertler A, Pearce W. A prospective evaluation of benzocaine-associated methemoglobinemia in human beings. Ann Emerg Med 1994;24:626-30.

2. O'Donohue W, Moss L, Angelillo V. Acute methemoglobinemia induced by topical benzocaine and lidocaine. Arch Intern Med 1980;140:1508-9.

3. Curry S. Methemoglobinemia. Ann Emerg Med 1979;11:21421.

4. Barker S, Tremper K, Hyatt J. Effects of methemoglobinemia on pulse oximetry and mixed venous oximetry. Anesthesiology 1989;70:112-7.

\title{
PULMONARY THROMBOENDARTERECTOMY FOR THE TREATMENT OF PULMONARY EMBOLISM CAUSED BY RENAL CELL CARCINOMA
}

\author{
Patrick Paw, MD, and Stuart W. Jamieson MB, FRCS, San Diego, Calif.
}

Chronic pulmonary embolism with pulmonary hypertension is optimally treated by bilateral pulmonary thromboendarterectomy with the use of cardiopulmonary bypass and profound hypothermic circulatory arrest. ${ }^{1}$ With very few exceptions, chronic pulmonary embolism is a bilateral disease. However, we recently performed a unilateral pulmonary thromboendarterectomy with exploration of the contralateral side for a patient with a chronic

From the Division of Cardiothoracic Surgery, University of California San Diego Medical Center, 200 West Arbor Dr., San Diego, Calif.

Received for publication Dec. 2, 1996; accepted for publication Jan. 23, 1997.

Address for reprints: Stuart W. Jamieson, FRCS, Department of Cardiothoracic Surgery, UCSD Medical Center, 200 West Arbor Dr., No. 8892, San Diego, CA 92103-8892.

J Thorac Cardiovase Surg 1997;114:295-7

Copyright (c) 1997 by Mosby-Year Book, Inc.

$0022-5223 / 97 \$ 5.00+0 \quad \mathbf{1 2 / 5 4 / 8 0 7 3 7}$ pulmonary embolus after a right nephrectomy for renal cell carcinoma.

Clinical summary. The patient is a previously healthy 40-year-old man who was referred for the evaluation and treatment of pulmonary hypertension. Three months earlier he had had chest pain and severe dyspnea on exertion. A ventilation/perfusion scan revealed the absence of perfusion to the entire left lung, with a few areas of subsegmental defects in the right lower lobe. He was initially treated with intravenous streptokinase, but this was discontinued because of a toxic reaction. After systemic anticoagulation, a computed tomographic scan of the abdomen and pelvis revealed a large renal tumor impinging on the inferior vena cava. A cardiac echocardiogram demonstrated normal left ventricular wall function with no evidence of a right atrial or ventricular mass, a lower extremity duplex scan showed no deep venous thrombosis, magnetic resonance imaging of the head showed no abnormalities, and results of a bone scan were negative.

A right nephrectomy was performed. Pathologic examination showed a renal cell carcinoma with invasion into 